Supporting Information

\title{
Spinel Cobalt Titanium Binary Oxide as an All-Non-Precious Water Oxidation Electrocatalyst in Acid
}

\author{
Sengeni Anantharaj, ${ }^{[a],[b]}$ Kannimuthu Karthick, ${ }^{[a],[b]}$ and Subrata Kundu ${ }^{*[a],[b]}$ \\ [a]Academy of Scientific and Innovative Research (AcSIR), Ghaziabad-201002, Uttar Pradesh, \\ India. \\ ${ }^{[b]}$ Materials Electrochemistry Division (MED), CSIR-Central Electrochemical Research Institute \\ (CECRI), Karaikudi-630006, Tamil Nadu, India. \\ * To whom correspondence should be addressed, E-mail: skundu@cecri.res.in; \\ kundu.subrata@gmail.com, Phone: (+ 91) 4565-241486 and (+91) 4565-241487.
}




\section{EXPERIMENTAL}

\section{Reagents and Instruments}

Cobalt (II) chloride, Titanium (III) Chloride in $\mathrm{HCl}$, Iridium oxide and potassium hydroxide were procured from Sigma-Aldrich. Sulphuric acid was purchased from RANKEM industries. $\mathrm{Hg} / \mathrm{HgO}$ reference electrode was purchased from $\mathrm{CH}$ Intruments Pvt. Ltd. Deionized water (18 M $\Omega$ ) was used for the entire synthesis and electrocatalysis processes. The synthesized spinel $\mathrm{Co}_{2} \mathrm{TiO}_{4}$ was characterized with HR-TEM, (Tecnai ${ }^{\mathrm{TM}} \mathrm{G}^{2}$ TF20) working at an accelerating voltage of $200 \mathrm{kV}$. The XRD analysis was done with a scanning rate of $5^{\circ} \mathrm{min}^{-1}$ in the $2 \theta$ range $10-90^{\circ}$ using a Bruker X-ray powder diffractometer (XRD) with $\mathrm{Cu} \mathrm{K}_{\alpha}$ radiation $(\lambda=0.154 \mathrm{~nm})$. X-ray photoelectron spectroscopic (XPS) analysis was performed using a Theta Probe AR-XPS system (Thermo Fisher Scientific, UK). The spinel $\mathrm{Co}_{2} \mathrm{TiO}_{4}$ modified carbon cloth (CC) was used as working electrode and a blank carbon cloth with large geometrical surface area $(1.5 \times 3$ $\mathrm{cm}$ ) was used as a counter electrode. $\mathrm{CH}$ Instrument model number $\mathrm{CHI} 6804 \mathrm{c}$ was used for entire electrochemical studies. 


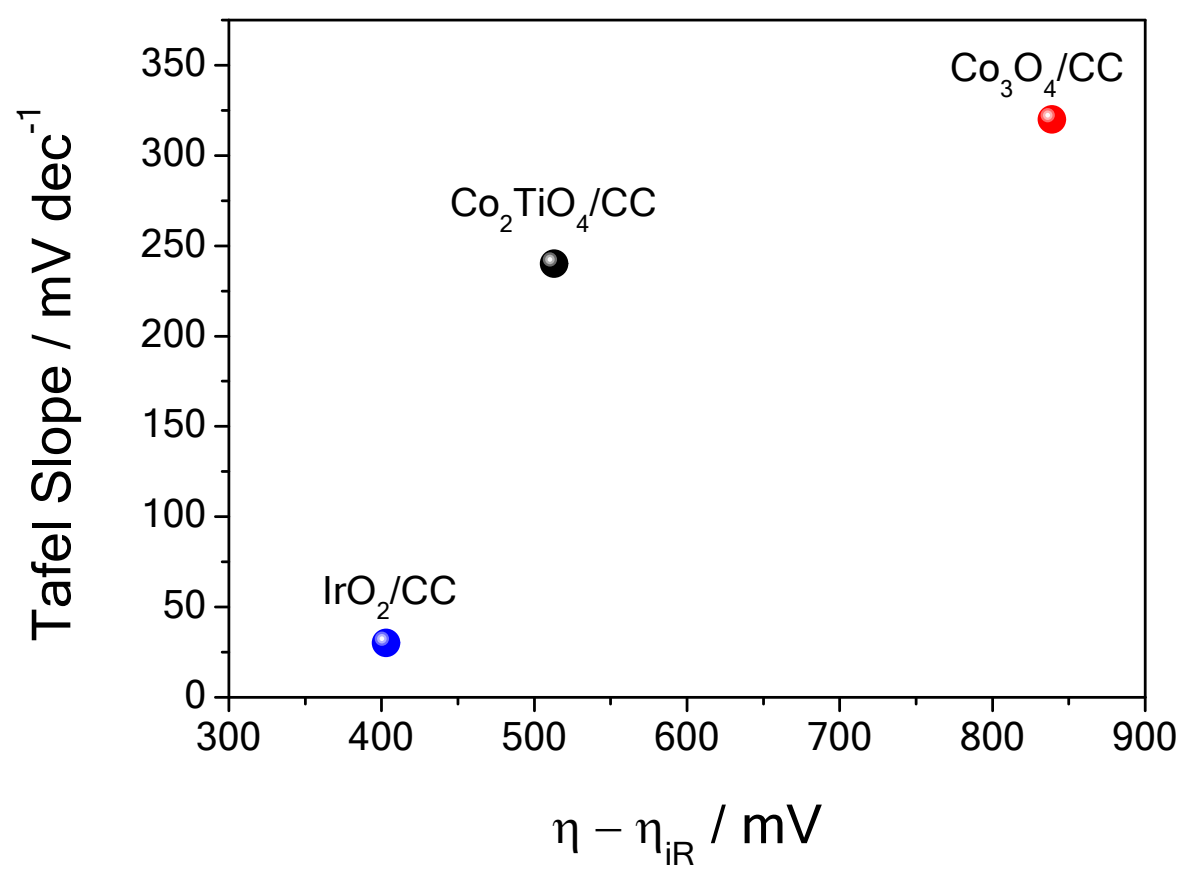

Fig. S1: Plot of overpotential vs. Tafel slope of $\mathrm{Co}_{2} \mathrm{TiO}_{4} / \mathrm{CC}$ interface in $0.5 \mathrm{M} \mathrm{H}_{2} \mathrm{SO}_{4}$. 

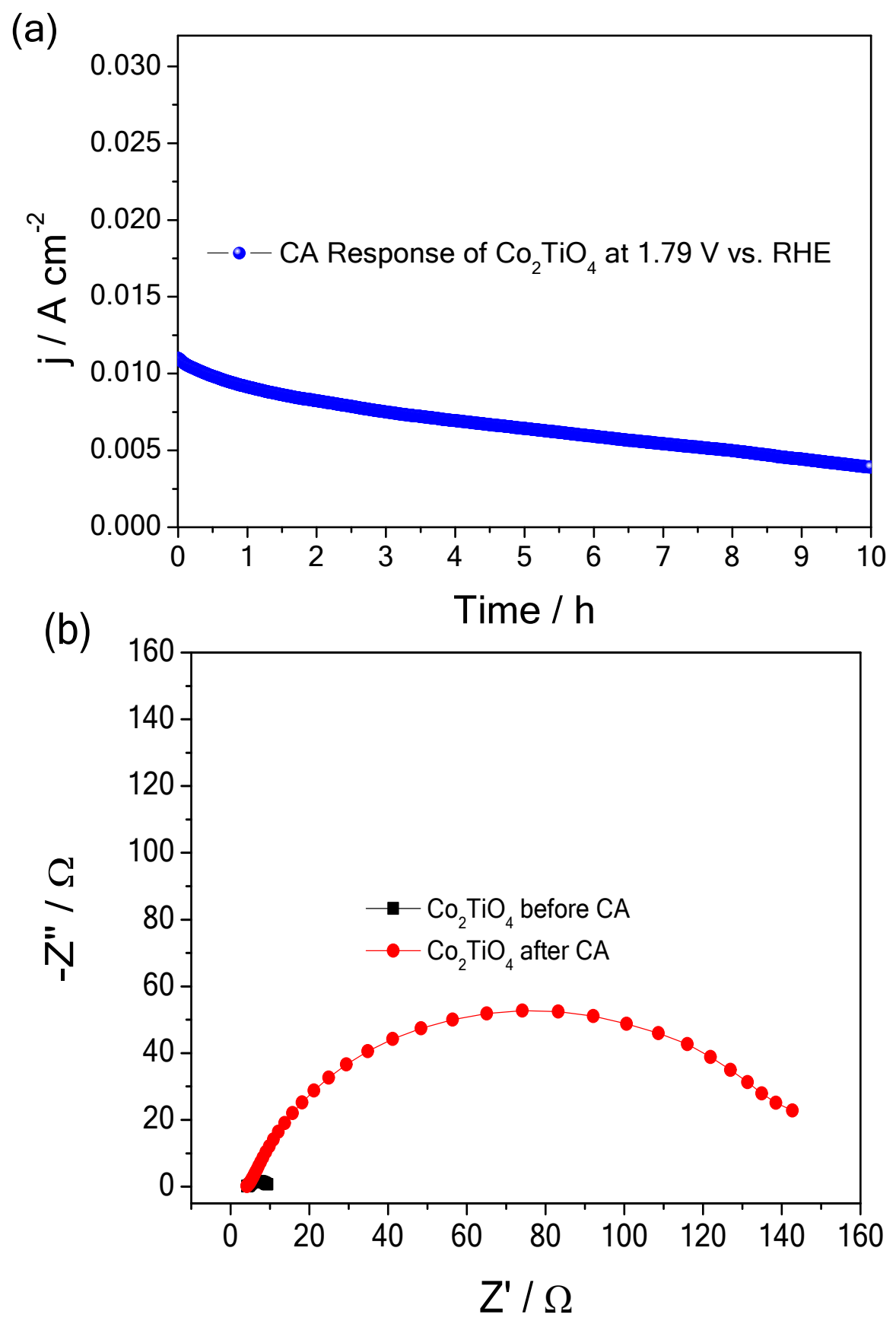

Fig S2: (a) Chronoamperometric response of $\mathrm{Co}_{2} \mathrm{TiO}_{4} / \mathrm{CC}$ at $1.79 \mathrm{~V}$ vs. RHE. (b) The Nyquist plots of the same before and after chronoamperometric analysis. 


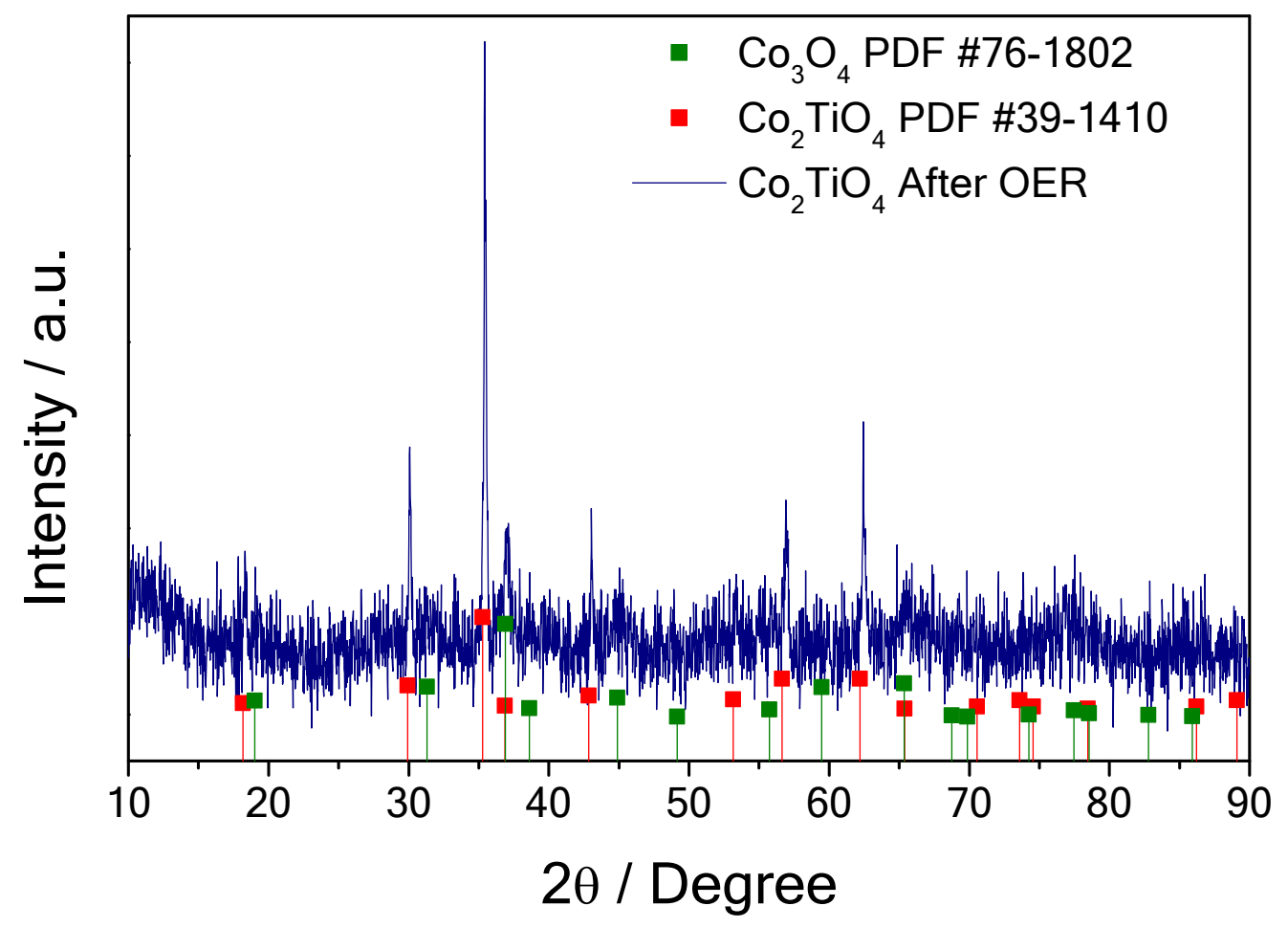

Fig. S3: XRD pattern of $\mathrm{Co}_{2} \mathrm{TiO}_{4}$ after OER studies showing the presence of $\mathrm{Co}_{3} \mathrm{O}_{4}$ along with $\mathrm{Co}_{2} \mathrm{TiO}_{4}$. 
(a)

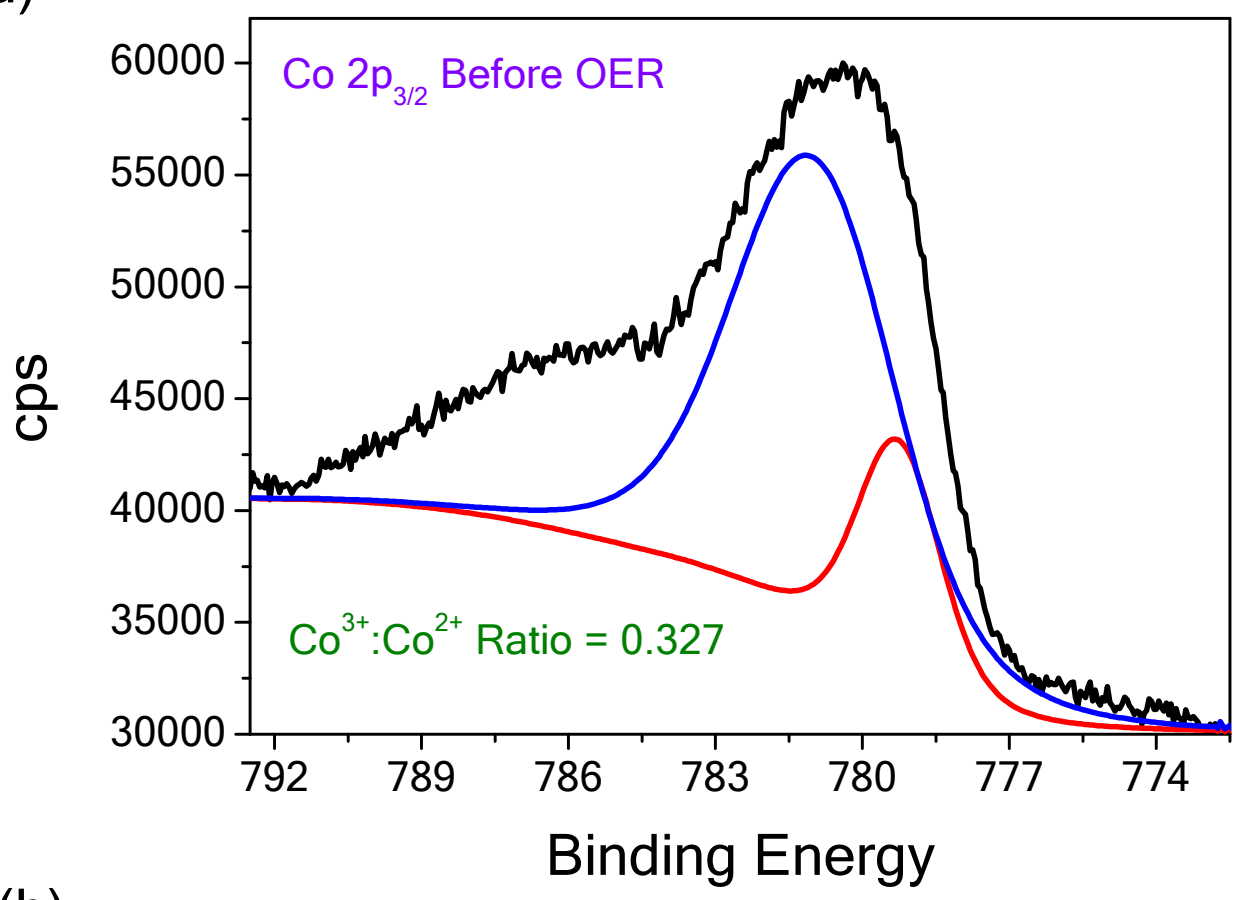

(b)

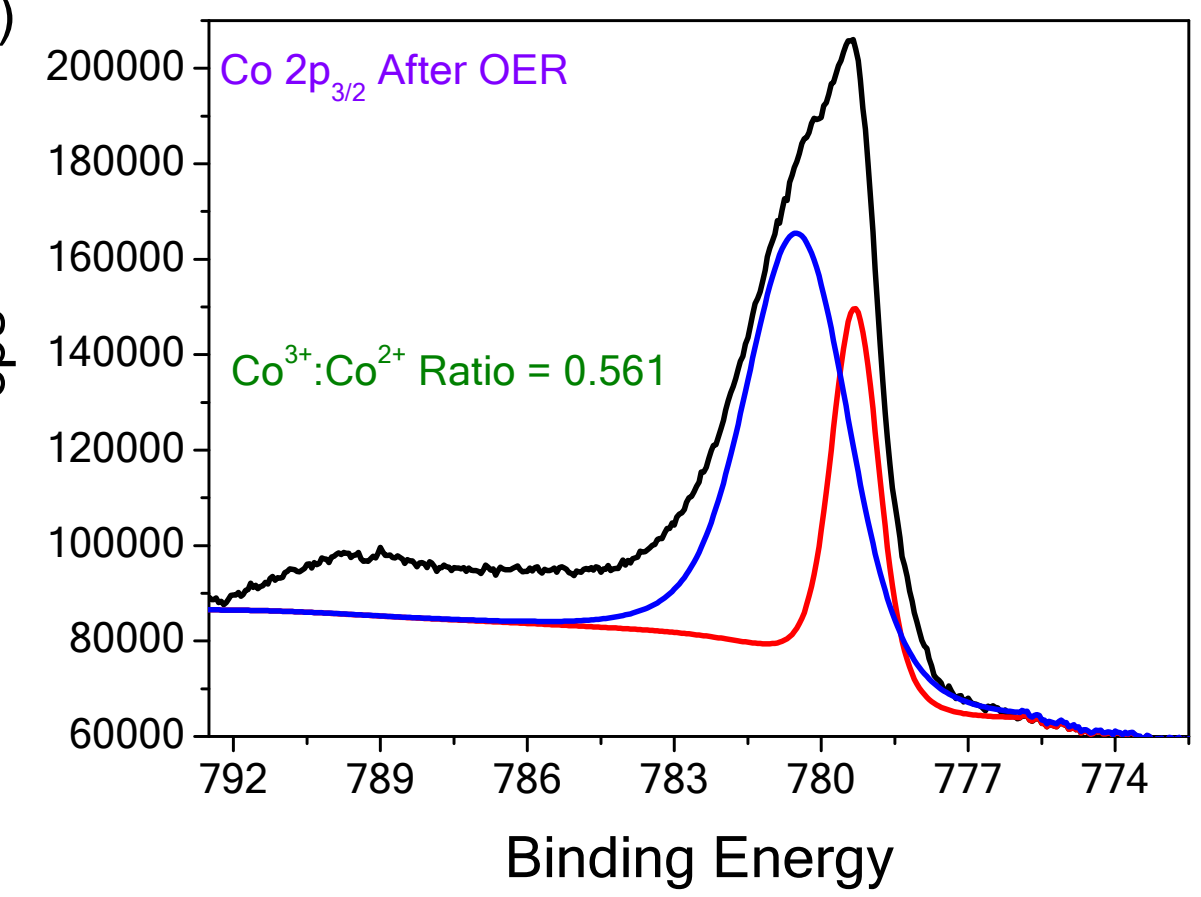

Fig. S4: (a-b) High resolution XPS spectra of Co 2p3/2 state of $\mathrm{Co}_{2} \mathrm{TiO}_{4}$ before and after OER studies. 